\title{
Dscam mutation leads to hydrocephalus and decreased motor function
}

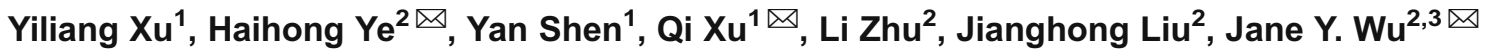 \\ ${ }^{1}$ National Laboratory of Medical Molecular Biology, Institute of Basic Medical Sciences, Chinese Academy of Medical Science \\ and Peking Union Medical College, Tsinghua University, Beijing 100084, China \\ 2 The State Key Laboratory of Brain and Cognitive Science, Institute of Biophysics, Chinese Academy of Sciences, \\ Beijing 100101, China \\ ${ }^{3}$ Department of Neurology, Center of Genetic Medicine, Lurie Cancer Center, Northwestern University Feinberg School of \\ Medicine, Chicago, IL 60611, USA \\ $\bowtie$ Correspondence: yehaihong@gmail.com (H. Ye), qixuangela@gmail.com (Q. Xu), jane-wu@northwestern.edu (J. Wu) \\ Received May 22, 2011 Accepted May 30, 2011
}

\begin{abstract}
The nervous system is one of the most complicated organ systems in invertebrates and vertebrates. Down syndrome cell adhesion molecule (DSCAM) of the immunoglobulin (Ig) superfamily is expressed widely in the nervous system during embryonic development. Previous studies in Drosophila suggest that Dscam plays important roles in neural development including axon branching, dendritic tiling and cell spacing. However, the function of the mammalian DSCAM gene in the formation of the nervous system remains unclear. Here, we show that $D s c a m^{d e / 17}$ mutant mice exhibit severe hydrocephalus, decreased motor function and impaired motor learning ability. Our data indicate that the mammalian DSCAM gene is critical for the formation of the central nervous system.
\end{abstract}

KEYWORDS Down syndrome cell adhesion molecule, motor function, motor learning, hydrocephalus

\section{INTRODUCTION}

The DSCAM gene is located on the human chromosome band 21q22.2-22.3, a critical region in Down syndrome (Yamakawa et al, 1998). The DSCAM gene encodes a transmembrane protein of the immunoglobulin $(\mathrm{lg})$ superfamily of neural cell adhesion molecules highly similar to L1CAM (Yamakawa et al., 1998). DSCAM consists of an ectodomain containing $10 \mathrm{lg}$ motifs and 6 fibronectin type III (FNIII) motifs, a transmembrane domain and a short intracellular domain (Yamakawa et al., 1998; Agarwala et al., 2000; Schmucker et al., 2000). As compared with the vertebrate DSCAM gene, the Drosophila Dscam gene has been studied in greater details. The Drosophila Dscam gene utilizes alternative splicing to produce more than 38,000 protein isoforms with distinct variant Ig domains located in the extracellular region (Ig2, Ig3, and Ig7) (Schmucker et al., 2000; Wojtowicz et al., 2004; Brites et al., 2008). Different Drosophila Dscam protein isoforms form homophilic dimers through perfectly matched $\lg 2$, Ig3 and $\lg 7$ domains (Agarwala et al., 2000; Wojtowicz et al., 2004; Hattori et al., 2007; Meijers et al., 2007; Wojtowicz et al., 2007). Drosophila Dscam plays an important role in axon targeting (Schmucker et al., 2000; Hummel et al., 2003), axon branching (Wang et al., 2002), dendritic self-avoidance and dendritic tiling (Hughes et al., 2007; Matthews et al., 2007; Soba et al., 2007). In vertebrates, DSCAM gene also plays a role in the nervous system development by affecting dendritic tiling, although the vertebrate DSCAM gene does not use extensive alternative splicing to generate tremendous numbers of DSCAM isoforms (Fuerst et al., 2008, 2009). Recent studies show that DSCAM acts as a receptor for Netrin and that DSCAM mediates commissural axon path-finding in chicken and mouse embryos (Ly et al., 2008; Liu et al., 2009). The mouse Dscam gene is expressed extensively in multiple regions of the developing and adult nervous system, including the choroid plexus, lateral ventricles, frontal cortex in embryos and the cortex, hippocampus, cerebellum as well as spinal cord (Agarwala et al., 2001; Barlow et al., 2002a). However, the biological function and possible involvement of the DSCAM gene in human pathogenesis remain unclear. In this study, we show that homozygous $D s c a m^{\text {del17 }}$ mutant mice develop severe hydrocephalus with dilated lateral 
ventricles, reduced thickness of cortex and corpus callosum, as well as deformed hippocampus. In addition, $D s$ am $^{\text {del17 }}$ mutation leads to decreased motor function and motor learning in mice. Our data uncover a previously unknown function of the mammalian Dscam gene in the formation of the central nervous system and in the pathogenesis of hydrocephalus.

Although further studies are necessary to understand the precise molecular mechanisms underlying hydrocephalus caused by $D s c a m^{\text {del17 }}$ mutation, our results indicate that Dscam is a new candidate gene of hydrocephalus. The $D$ scam ${ }^{\text {del17 }}$ mutant mice represent a new animal model for hydrocephalus.

\section{RESULTS}

\section{Dscam ${ }^{\text {del17 }}$ mutation leads to retarded growth and impaired motor coordination}

The $D s c a m^{\text {del17 }}$ mutation is a 38 bp deletion in exon 17 of the Dscam gene, resulting in a reading-frame shift and a truncated DSCAM protein. Thus, the mutant DSCAM gene is predicted to form a protein truncated at the second FNIII motif in the extracellular domain (Fig. 1A). Dscam mRNA levels in the brain were also reduced by approximately $70 \%$ in $D_{\text {scam }}{ }^{\text {del17 }}$ mutant mice (Fuerst et al., 2008). Using PCR with the mouse Dscam gene specific primers, we could differentiate wild-type $(\mathrm{Wt})$ mice that produced a $170 \mathrm{bp}$ band from homozygous $D$ scam ${ }^{\text {del17 }}$ mutant $(\mathrm{hm})$ that was detected as a $133 \mathrm{bp}$ band, with both bands observed in heterozygous mutant (het) (Fig. 1B). Previous data show that homozygous $D$ scam $^{\text {del17 }}$ mutant mice on C57BJ/6 background died soon after birth. However, the homozygous $D_{s c a m}{ }^{\text {del17 }}$ mutant mice can survive to adulthood on C57BJ/6 and BALB/C congenic background (Fuerst et al., 2008; Amano et al., 2009). To study the function of Dscam gene at postnatal stages in mice, $D$ scam ${ }^{\text {del17 }}$ homozygous mutant mice were analyzed on C57BJ/6 and BALB/C congenic background. Although $D s c a m^{\text {del17 }}$ homozygous mutant mice were indistinguishable from their wild type littermates at birth, $D_{\text {scam }}{ }^{\text {del17 }}$ homozygous mutant mice gradually became uncoordinated during the first 3 weeks after birth. By the age of 3 weeks, Dscam ${ }^{\text {del17 }}$ homozygous mutant mice showed reduced body sizes with dome-shaped heads, tiny ears and often closed eyes (Fig. 1C). Gross anatomical examinations reveal that $D s c a m^{d e / 17}$ homozygous mutant mice had abnormal dome-shaped cranial cavities (Fig. 1D). These Dscam mutant mice also showed a walking posture that was remarkably different from that of their wild type littermates at the same age (see Supplementary videos). Nonetheless, Dscam ${ }^{\text {del17 }}$ homozygous mutant mice eventually caught up in their growth, with their body sizes similar to those of wild type littermates by the age of two months although the Dscam mutant mice retained their peculiar walking posture. Such Dscam mutant mice stood on their toes

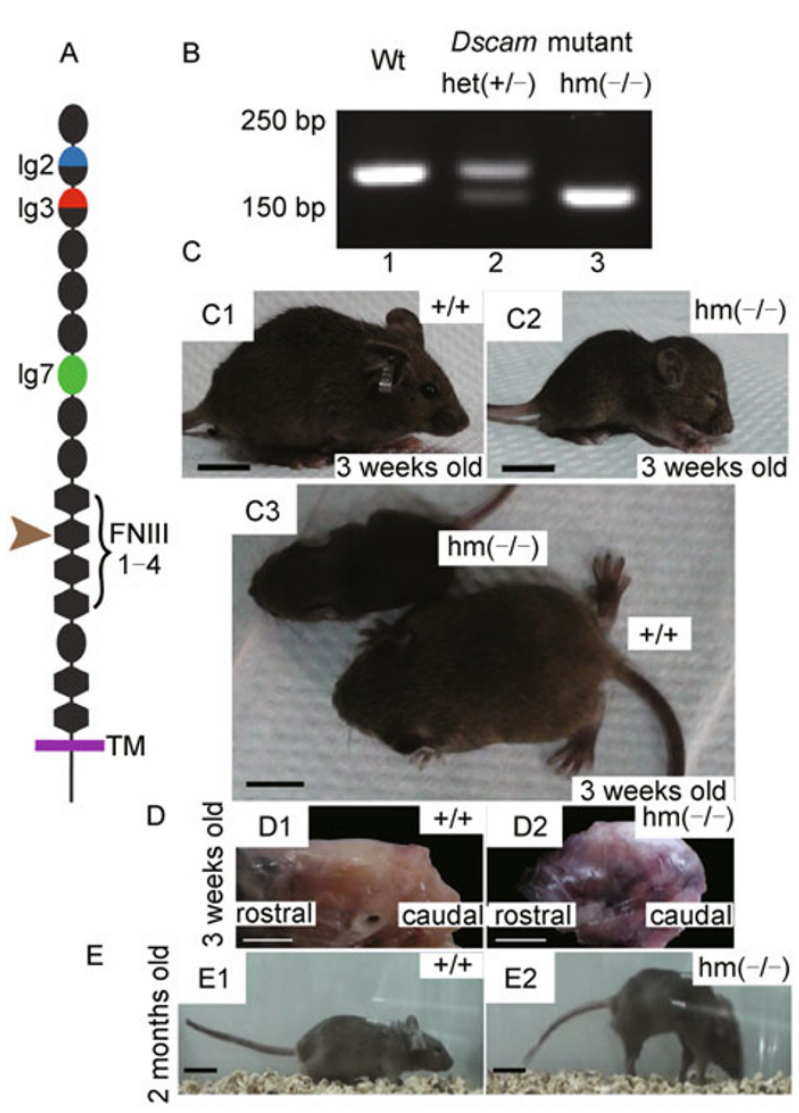

Figure 1. Abnormal body size, head shape and walking posture of homozygous Dscam $^{\text {del17 }}$ mutant mice. (A) The domain structure of DSCAM. The Dscam ${ }^{\text {del17 }}$ mutation results in a truncated protein at the second FNIII domain. (B) Genotyping of $D$ scam ${ }^{\text {del17 }}$ mutant mice. PCR with the specific primers led to detection of a $170 \mathrm{bp}$ band in wild type mice (panel 1), a $133 \mathrm{bp}$ band in homozygous Dscam de/17 mutant mice (panel 3), and both bands in heterozygous $D$ scam ${ }^{\text {del17 }}$ mutant mice (panel 2). (C) Physical characteristics of $D s c a m^{\text {del17 }}$ mutant mice. At 3 weeks old, homozygous $D s c a m^{\text {del17 }}$ mutant mice show delayed development, exhibiting smaller body size (C3), dome-shaped head, small ears and closed eyes (C2) as compared with their wild type littermates (C1). Moreover, at this age, wild type mice were able to walk whereas homozygous $D_{\text {scam }}{ }^{\text {del17 }}$ mutant mice were not (scale bar: $1 \mathrm{~cm}$ ) (D) Dome-shaped head of a homozygous Dscam del17 mutant mouse (D1) as compared with that of the wild type mouse (D2) (scale bar: $0.5 \mathrm{~cm}$ ) (E) Change in locomotion. The body sizes of homozygous Dscam del17 mutant mice gradually increased and became similar to that of wild type mice. By 2 months of age, homozygous Dscam del17 mutant mice (E1) were almost the same as the wild type mice in size (E2). As compared with wild type mice (E2), homozygous Dscam de/17 mutant mice showed abnormal locomotion by walking on their toes with tetanic hind limbs bent back and down-curved tails (E1) (scale bar: $2 \mathrm{~cm}$ ) [Wt, wild type; het(+/-), heterozygous Dscam del17 mutant mice; hm(-l-), homozygous Dscam ${ }^{\text {del17 }}$ mutant mice]. 
and walked with tetanic hind limbs bent back, putting down their forelimbs and curving their tails downward to balance their bodies (Fig. 1E). On the other hand, Dscam del17 heterozygous mutant mice did not show obvious differences from their wild type littermates in either their body sizes or the walking posture.

\section{Dscam ${ }^{\text {del17 }}$ mutation leads to decreased motor function and motor learning ability}

The peculiar walking posture of Dscam ${ }^{\text {del17 }}$ homozygous mutant mice prompted us to examine their motor function using the classic rotarod test (Jones and Roberts, 1968). At 0 time point, mice of different genotypes, wild type, heterozygous or homozygous $D s c a m^{\text {del17 }}$ mutant, were placed on the rod (Fig. 2A1). Thirty-five seconds after spinning of the rotarod began, the $D s c a m^{\text {del17 }}$ homozygous mutant mice fell off the rotarod (at the speed of $15.5 \mathrm{rpm}$ ) (Fig. 2A2), whereas heterozygous $D s c a m^{\text {del17 }}$ mutant mice did not fall off until 102 seconds after the rotarod began to spin and reached the speed of $36.3 \mathrm{rpm}$ (Fig. 2A3). The wild type littermates stayed on the rotarod until the speed reached $40 \mathrm{rpm}$ (Fig. 2A). Consistent results were obtained in independent experiments. Dscam ${ }^{\text {del17 }}$ homozygous mutant mice $(n=10)$ failed to stand steadily even on the resting rod and fell off within a short period time $(28.30 \mathrm{~s} \pm 5.54 \mathrm{~s})$, whereas the average rotarod running time of wild type mice ( $n=6,115.70 \mathrm{~s} \pm 23.33 \mathrm{~s})$ or heterozygous $D s c a m^{\text {del17 }}$ mutant mice $(n=5,132.00 \mathrm{~s} \pm$ $24.39 \mathrm{~s}$ ) were significantly longer (Fig. 2B). As compared with wild type mice $(n=6,33.67 \mathrm{rpm} \pm 2.21 \mathrm{rpm})$ or heterozygous $D_{\text {scam }}{ }^{\text {del17 }}$ mutant mice $(n=5,35.59 \mathrm{rpm} \pm 2.16 \mathrm{rpm})$ mice, homozygous $D$ scam ${ }^{\text {del17 }}$ mutant mice $(n=6,14.73 \mathrm{rpm} \pm$ $2.22 \mathrm{rpm}$ ) fell off the rotarod at lower speeds (Fig. 2C). The differences between the wild type and heterozygous $D_{\text {scam }}{ }^{\text {del17 }}$ mutant mice were not statistically significant. These data support that homozygous $D s c a m^{\text {del17 }}$ mutant mice have impaired motor coordination.

Advanced rotarod training was carried out to assess the motor learning ability of $D s c a m^{d e / 17}$ mutant mice. Mice were trained for 8 trials in two days. After 8 trials, the average of running time of the wild type mice $(n=4)$ was significantly extended (1st: $84.50 \mathrm{~s} \pm 23.29 \mathrm{~s}$, 8th: $188.80 \mathrm{~s} \pm 29.59 \mathrm{~s}$ ). However, the average running time of homozygous $D_{\text {scam }}{ }^{\text {del17 }}$ mutant mice $(n=3)$ showed no significant change following eight training sessions: $1 \mathrm{st}: 8.00 \mathrm{~s} \pm 2.89 \mathrm{~s}$ and 8th: $18.33 \mathrm{~s} \pm 10.87 \mathrm{~s}$. Heterozygous $D s c a m^{d e 117}$ mutant mice $(n=$ 5) showed similar phenotype as the wild type mice: 1st: $111.80 \mathrm{~s} \pm 30.08 \mathrm{~s}$ and 8 th: $187.60 \mathrm{~s} \pm 23.42 \mathrm{~s}$ (Fig. 2D). These results revealed the impaired motor learning ability of Dscam ${ }^{\text {del17 }}$ mutant mice.

\section{$D s c a m^{d e / 17}$ mutation leads to severe hydrocephalus}

Decreased motor coordination and impaired motor learning ability of $D s c a m^{\text {del17 }}$ mutant mice prompted us to test the hypothesis that the Dscam mutation may affect the formation and/or function of the central nervous system in mice. The $D s c a m^{\text {del17 }}$ mutant mice together with their littermate controls were examined at the age of two months. Two of five homozygous Dscam ${ }^{\text {del17 }}$ mutant mice showed remarkable changes in their brains with obvious deformation, including collapsed cortex in the lateral-caudal region and reduced sizes of the olfactory bulbs (Fig. 3A1 and 3A2). There were variations in the brain malformation among individual $D$ scam mutant mice with some showing severe deformation in the middle to caudal regions (Fig. $3 A 3$ and $3 A 4$ ). To further characterize the brain phenotype among these Dscam mutant mice, we carried out Nissl staining using serial brain sections of $D s c a m^{d e l 17}$ mutant mice and compared them with age-matched wild type and heterozygous mutant littermates (Fig. 3B). All of homozygous Dscam ${ }^{\text {del17 }}$ mutant brains exhibited obvious ventricular dilation, although severities and the affected regions varied among different individuals (Fig. 3B3, B7, B11, B15, B4, B8, B12, and B16). Although there were variations in severity, enlargement of lateral ventricles was observed in all of homozygous Dscam mutant brains. Three of five mice exhibited lateral ventricles that were merged into one large ventricle and developed new ventricles located on the lateral of striatum on both sides (Fig. 3B4 and B8). In addition to dilation of the dorsal third ventricle and the third ventricle, the aqueduct connecting the third and fourth ventricle was also enlarged (Fig. 3B). Quantification data shows that compared with wild type $\left(n=4,3.83 \mathrm{~mm}^{3} \pm\right.$ $\left.0.33 \mathrm{~mm}^{3}\right)$ and heterozygous $D s c a m^{\text {del17 }}$ mutant mice $(n=3$, $2.55 \mathrm{~mm}^{3} \pm 0.28 \mathrm{~mm}^{3}$ ) mice, the ratio of ventricular volume to brain of homozygous Dscam ${ }^{\text {del17 }}$ mutant mice $(n=5,20.36$ $\mathrm{mm}^{3} \pm 4.30 \mathrm{~mm}^{3}$ ) mice brains was significantly enlarged. Moreover, the ventricular volume of homozygous $D_{s c a m}{ }^{\text {del17 }}$ mutant mice $\left(n=5,49.79 \mathrm{~mm}^{3} \pm 13.97 \mathrm{~mm}^{3}\right)$ was also significantly larger than wild type $\left(n=4,7.64 \mathrm{~mm}^{3} \pm\right.$ $\left.0.88 \mathrm{~mm}^{3}\right)$ and heterozygous $D s c a m^{\text {del17 }}$ mutant mice $(n=$ $3,4.73 \mathrm{~mm}^{3} \pm 0.66 \mathrm{~mm}^{3}$ ) mice.

\section{Malformation of cortex, corpus callosum and commissural fibers in Dscam ${ }^{\text {del17 }}$ mutant mice}

Homozygous Dscam ${ }^{\text {del17 }}$ mutant brains showed abnormal shape, possibly as a consequence of ventricular dilation. Coronal sections of control and $D s c a m^{\text {del17 }}$ mutant brains are shown in Fig. 3. The hippocampus of homozygous $D_{\text {scam }}{ }^{\text {del17 }}$ mutant brains showed remarkable malformation (Fig. 3B12, B15, and B16). The striatum was constricted and internal capsule fibers were extended (Fig. 3B3, B4, B7, and B8). Nissl-staining of serial sections reveal that the cortex and commissural fibers were thinner and the corpus callosum failed to form properly with severe deformations especially in the caudal regions (Fig. 3B3, B7, B11, B15, B4, B8, B12, and B16). The phenotypes were observed in all of five homo- 
A
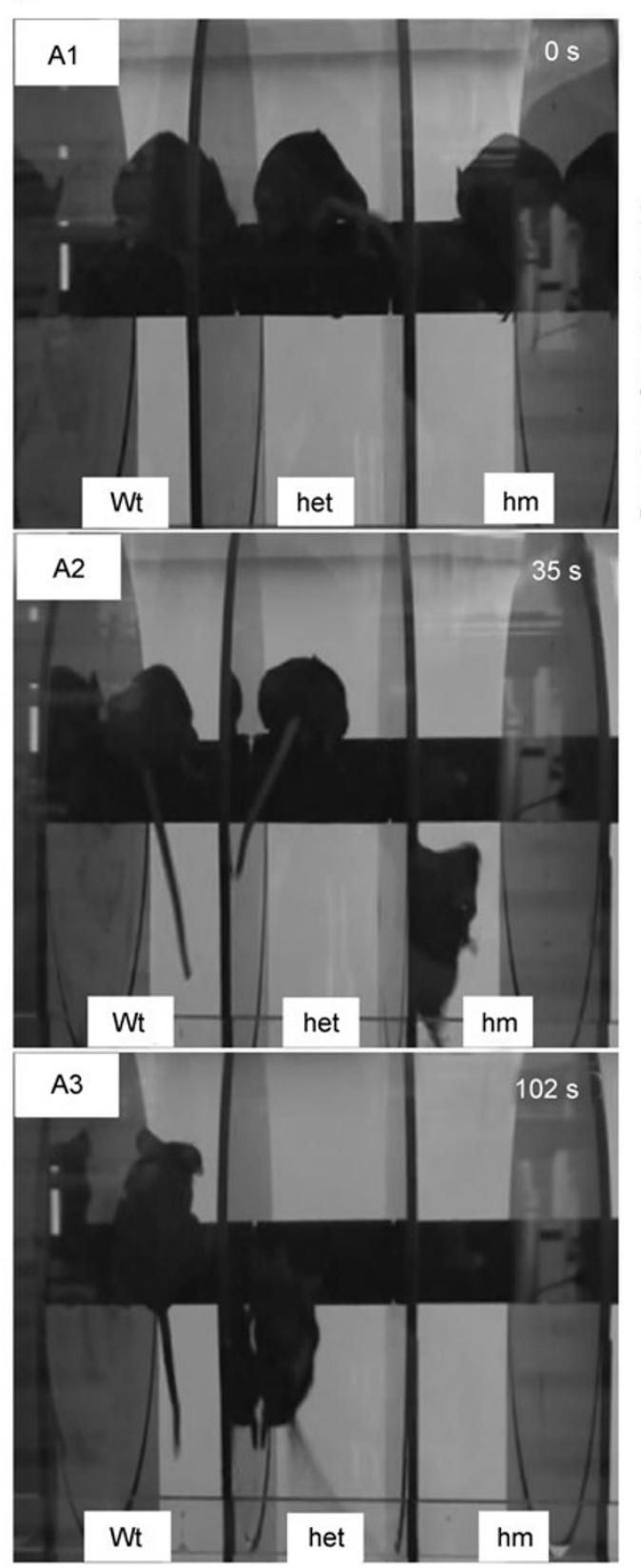

B

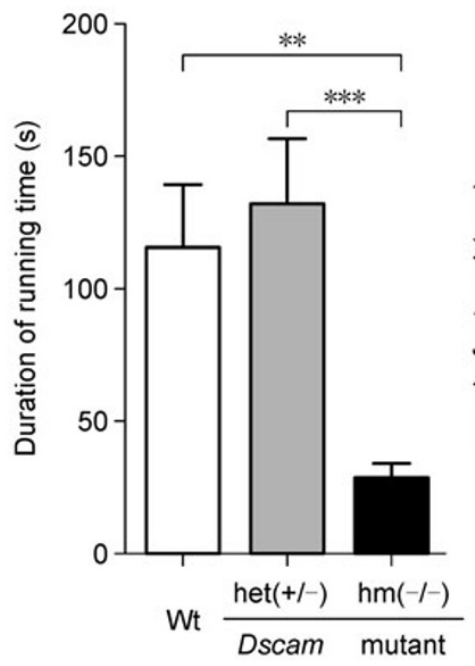

C

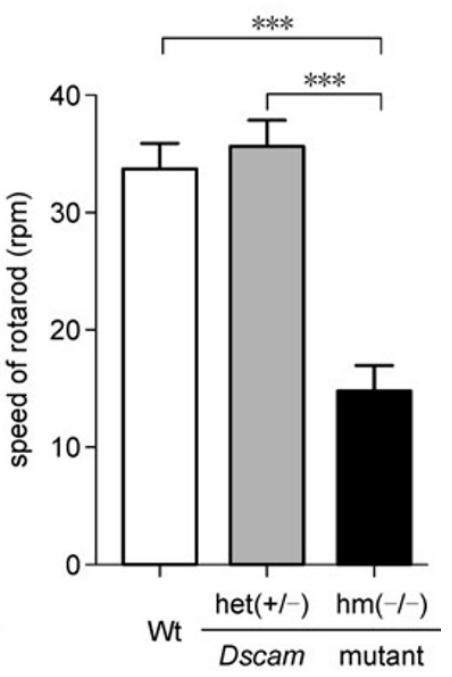

D

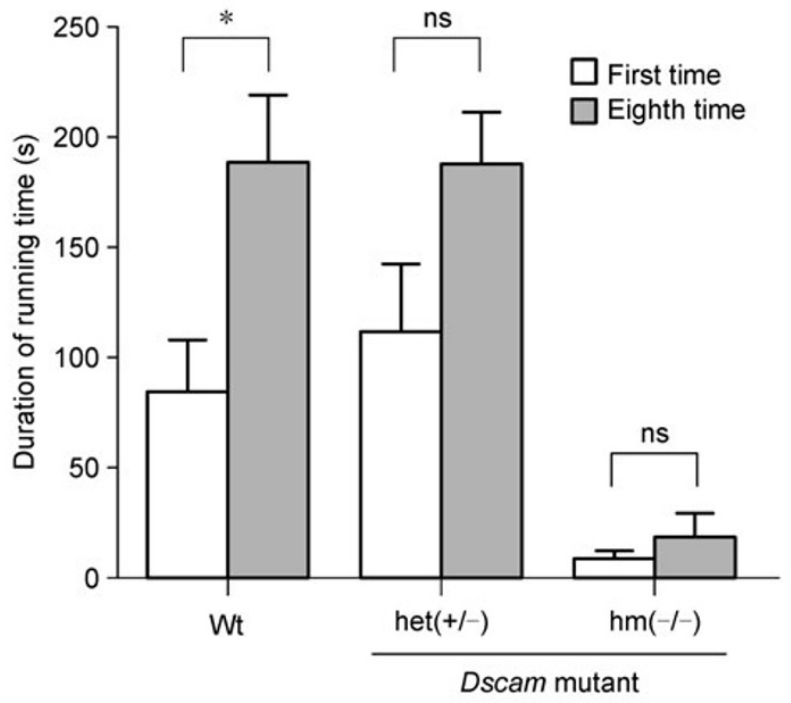

Figure 2. Impaired motor function and motor learning ability of homozygous Dscam ${ }^{\text {del17 }}$ mutant mice. (A) In the rotarod test, at $0 \mathrm{~s}$, three groups of mice with different genotypes were placed on the resting rod (A1). At $35 \mathrm{~s}$, homozygous $D s c a m^{\text {del17 }}$ mutant mice fell off (A2). At $102 \mathrm{~s}$, the heterozygous $D$ scam ${ }^{\text {del17 }}$ mutant mice fell off (A3). (B and C) Motor function as measured by the rotarod test. The average running duration on the rotarod of wild type and heterozygous $D s c a m^{\text {del17 }}$ mutant mice were significant longer than the homozygous $D s c a m^{\text {del17 }}$ mutant mice (means \pm SEM, One-way ANOVA and Bonferrioni's multiple comparison test, ${ }^{* *} p<0.01 ;{ }^{* * *} p<0.001$ ). As compared with the wild type and heterozygous $D s c a m^{\text {del17 }}$ mutant mice, homozygous $D s c a m^{\text {del17 }}$ mutant mice fell off at a lower speed (means \pm SEM, One-way ANOVA and Bonferrioni's multiple comparison test, ${ }^{* * *} p<0.001$ ). (D) The average running duration of mice after training. After training, the average running duration of the wild type mice was significantly extended, whereas that of heterozygous $D s c a m^{\text {del17 }}$ mutant mice and homozygous $D s c a m^{\text {del17 }}$ mutant mice had no significant difference before and after training (means \pm SEM, Two-way ANOVA and Bonferroni posttests, ${ }^{*} p<0.05$; ns, no significance) [Wt, wild type; het(+/-), heterozygous $D s c a m^{\text {del17 }}$ mutant mice; hm(-I-), homozygous Dscam ${ }^{\text {del17 }}$ mutant mice]. 


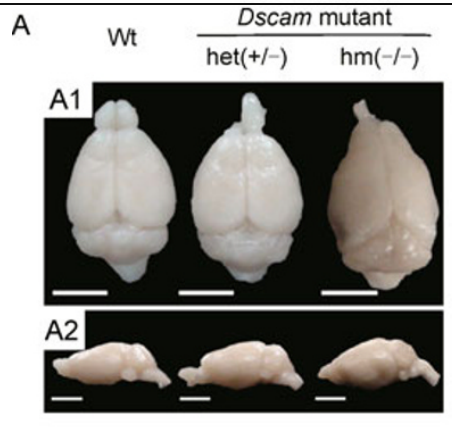

B $\mathrm{B}$

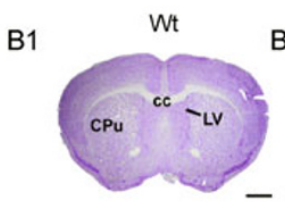

B5

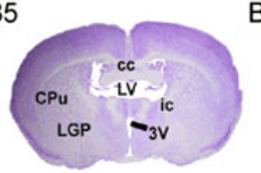

B2

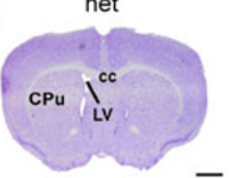

B6

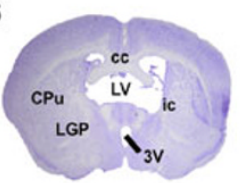

B9

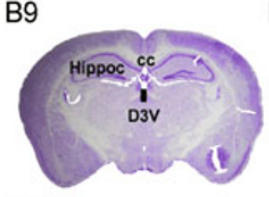

B13

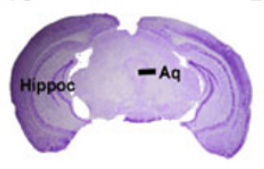

B10

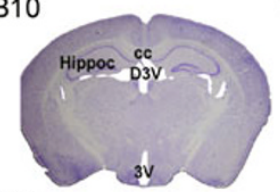

B14

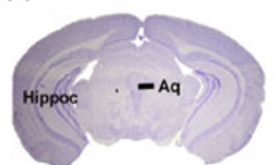

B3

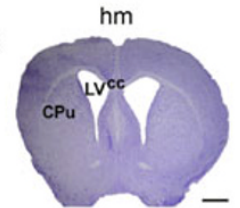

B7

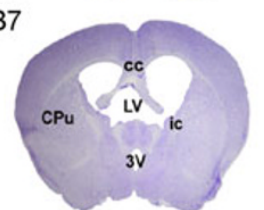

B11

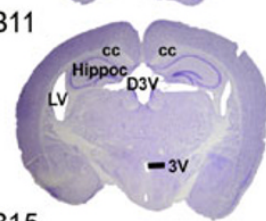

B17

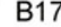

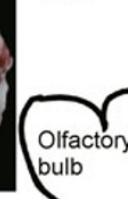

$x^{2} \leqslant 5^{6}$

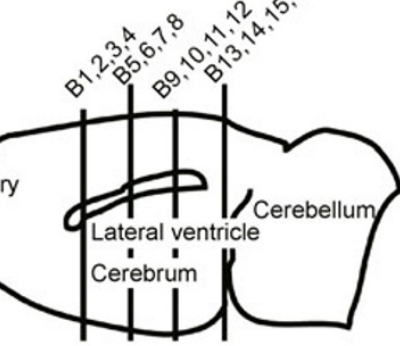

15

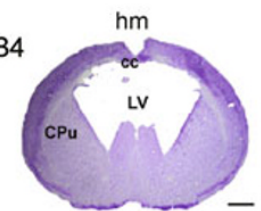

B8

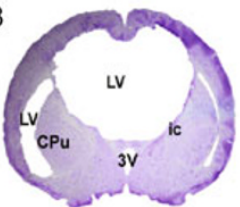

B12

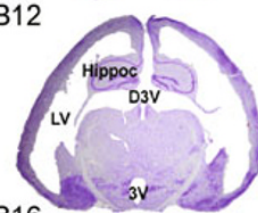

B16
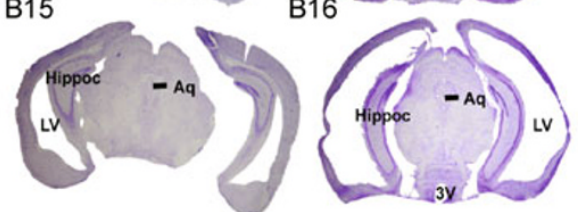

D

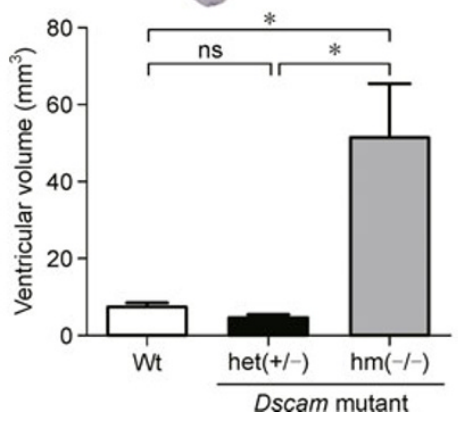

Figure 3. Hydrocephalus in homozygous Dscam del17 mutant mice. (A) From the dorsal (A1) and lateral (A2) views, homozygous Dscam del17 mutant mice brains show significant deformation with collapsed cortices in the caudal region and smaller olfactory bulbs. The degree of brain tissue collapse varies among different individual mice. Two out of five brains (A3) showed mild changes in the caudal region, whereas other three brains (A4) exhibited severe collapse from the middle to caudal regions (scale bars: $0.5 \mathrm{~cm}$ ). (B) Coronal sections of brains were stained using Nissl staining. Coronal section of the wild type mice (B1, B5, B9, and B13) and heterozygous Dscam del17 mutant mice (B2, B6, B10, and B14) showed normal ventricles, whereas age-matched homozygous Dscam del17 mutant mice (B3, B4, B7, B8, B11, B12, B15, and B16) exhibited enlarged cerebral ventricles, a progressively-thinned cortical mantle, and stretched internal capsule fibers at different rostrocaudal levels. Different homozygous $D_{\text {scam }}^{\text {del17 }}$ mutant mice showed different degrees of ventricular enlargement (B3, B4, B7, B8, B11, B12, B15, and B16). (B17) A diagram illustrating the mouse brain and positions of four different coronal sections (scale bar, $1 \mathrm{~mm}$; cc, corpus callosum; CPu, caudate putamen (striatum); LV, lateral ventricle; LGP, lateral globus pallidus; ic, internal capsule; 3V, 3rd ventricle; Hippoc, Hippocampus; D3V, drosal 3rd ventricle; Aq, aqueduct). (C and D) Comparison of ventricular size. Compared with wild type and heterozygous Dscam del17 mutant mice, homozygous Dscam del17 mutant mice showed significant increases in the ventricular volume, either measured by the ratio of ventricular to total brain volumes (panel C, means \pm SEM, One-way ANOVA and Bonferrioni's multiple comparison test, * $p<0.05$; ns, no significance) or as absolute ventricular volumes (panel D) [Wt, wild type; het $(+/-)$, heterozygous Dscam ${ }^{\text {del17 }}$ mutant mice; $\mathrm{hm}(-/-)$, homozygous Dscam ${ }^{\text {del17 }}$ mutant mice]. 
zygous $D s c a m^{\text {del17 }}$ mutant brains. Different cortical regions such as motor and sensory cortices of homozygous $D s c a m^{d e / 17}$ mutant mice were displaced laterally and became much thinner than wild type mice (Fig. 4A). The dorso-ventral thickness of the corpus callosum was much reduced not only in the medial region of the brain but also in the cortical region (Fig. 4B). The corpus callosum formed thinner commissure in the medial region of the brain, and appeared disrupted or even disappeared in caudal parts (Fig. 3B8, B11, B12, and B16). It is likely that such severe malformation of corpus callosum may cause disconnection of cortices.

\section{DISCUSSION}

Hydrocephalus, a common medical condition, is pathological dilation of cerebral ventricles, with the intracranial spaces filled with cerebrospinal fluid (CSF). Produced by the choroid plexus, CSF flows from the lateral ventricles into the third ventricle through the foramina of Monro, then down the aqueduct into the fourth ventricle, and finally, into the cistern magna through two lateral foramina and the single midline foramen. Abnormal accumulation of CSF may cause dilation of the cerebral ventricles and result in a gradual destruction of periventricular structures, including the corpus callosum and the fimbria-fornix pathway (Del Bigio, 2010). Therefore, clinical syndrome of hydrocephalus often manifests as brain dysfunction as a result of subcortical disconnection.

Hydrocephalus in human can be classified into two clinical forms: congenital and acquired. Often associated with congenital malformation of brain structures or brain tumor, infection or brain trauma, hydrocephalus may be caused by excessive production, reduced absorption or obstructed circulation of CSF. The molecular mechanisms underlying pathogenesis of hydrocephalus are largely unknown. Genetic studies show that hydrocephalus is a heterogeneous and multi-factorial disease. Approximately $40 \%$ of hydrocephalus cases have genetic components (Haverkamp et al., 1999). At least 43 genes have been associated with hydrocephalus, including 10 genes for congenital hydrocephalus in animal models and one X-linked hydrocephalus gene, L1CAM, in humans (Jouet et al., 1993; Basel-Vanagaite et al, 2006; Zhang et al., 2006; Liebau et al, 2007; Jackson et al, 2009; Wilson et al, 2009; Bertolin et al, 2010; Nakamura et al, 2010; Schäfer and Altevogt, 2010; Tapanes-Castillo et al, 2010; Vos and Hofstra, 2010).

Since its original discovery (Yamakawa et al., 1998), significant efforts have been made in studying mammalian DSCAM function (Agarwala et al, 2000, 2001; Barlow et al, 2002a, 2002b; Fuerst et al, 2008, 2009; Ly et al., 2008; Liu et al., 2009). Similar to sidekick and other neural adhesion molecules, DSCAM is a synaptic protein (see references in Yamagata and Sanes, 2010). Previous studies have reported the involvement of DSCAM in neural development, including axon guidance and branching, dendritic patterning and self- avoidance, axon target recognition and synaptic formation. Recent data suggest that DSCAM can act as a receptor for netrin-1, mediating pathfinding of commissural axons at the midline (Ly et al., 2008; Liu et al., 2009). However, molecular pathways mediating netrin-DSCAM signaling remain to be defined.

Analyses of $D s c a m^{\text {del17 }}$ mutant mice reveal that homozygous $D s c a m^{\text {del17 }}$ mutant mice exhibit delayed growth and impaired locomotive function. Advanced rotarod tests show that the mean running times of homozygous $D_{s c a m}{ }^{\text {del17 }}$ mutant mice were significantly shorter, and the speeds of rotarod when homozygous $D s c a m^{\text {del17 }}$ mutant mice fell off were significantly lower as compared with that observed among the wild type and heterozygous $D s c a m^{\text {de/17 }}$ mutant mice. Even after training, the running times of homozygous $D s c a m^{\text {del17 }}$ mutant mice were not significantly extended, suggesting decreased motor function and motor learning ability of homozygous $D s c a m^{\text {del17 }}$ mutant mice. The impairment in the motor ability may be explained by the malformation of the central nervous system in homozygous $D s c a m^{\text {del17 }}$ mutant mice. In the developing mouse nervous system, Dscam expression was first detected at E10.5 in the ventral neural tube, the source of motor neuroblast, and in motor neurons of the ventral neural tube by E11.5. By E12.5, Dscam expression was detected in the motor neurons of the ventral spinal cord (Barlow et al., 2002b). In the adult brain, Dscam was expressed in pyramidal cells in the cortex and in Purkinje cells of the cerebellum. The expression pattern of Dscam supports its role in the development and function of motor neurons.

In addition, homozygous $D s c a m^{\text {del17 }}$ mutant mice showed severe hydrocephalus with dilation in the ventricles system, deformed hippocampus, stretched internal capsule fibers and thinner cortex and corpus callosum. The dilation of the cerebral ventricles caused gradual destruction of white matter neurons and secondary changes of neuronal cell bodies and synapse (Del Bigio, 2010). However, the precise mechanisms are still unknown. Genetic factors are believed to play important roles in the pathogenesis of hydrocephalus. Previous research have characterized at least 43 mutants associated hydrocephalus, 10 of which were congenital hydrocephalus genes identified in animal models, and only one $\mathrm{X}$-linked hydrocephalus gene (L1CAM) has been identified in humans (Jouet et al., 1993; Zhang et al., 2006). The motor functional deficiency of Dscam mutant mice may result from severe hydrocephalus. Although the mechanism of hydrocephalus caused by $D s c a m^{d e l 17}$ mutation needs to be further explored, our study uncovers a previously unknown function of mammalian DSCAM gene in the formation of the central nervous system and in the pathogenesis of hydrocephalus. Our data not only suggest that Dscam is a new candidate gene for hydrocephalus, but also provide a new animal model for research on hydrocephalus. In our future studies, it will be interesting to investigate and compare the 

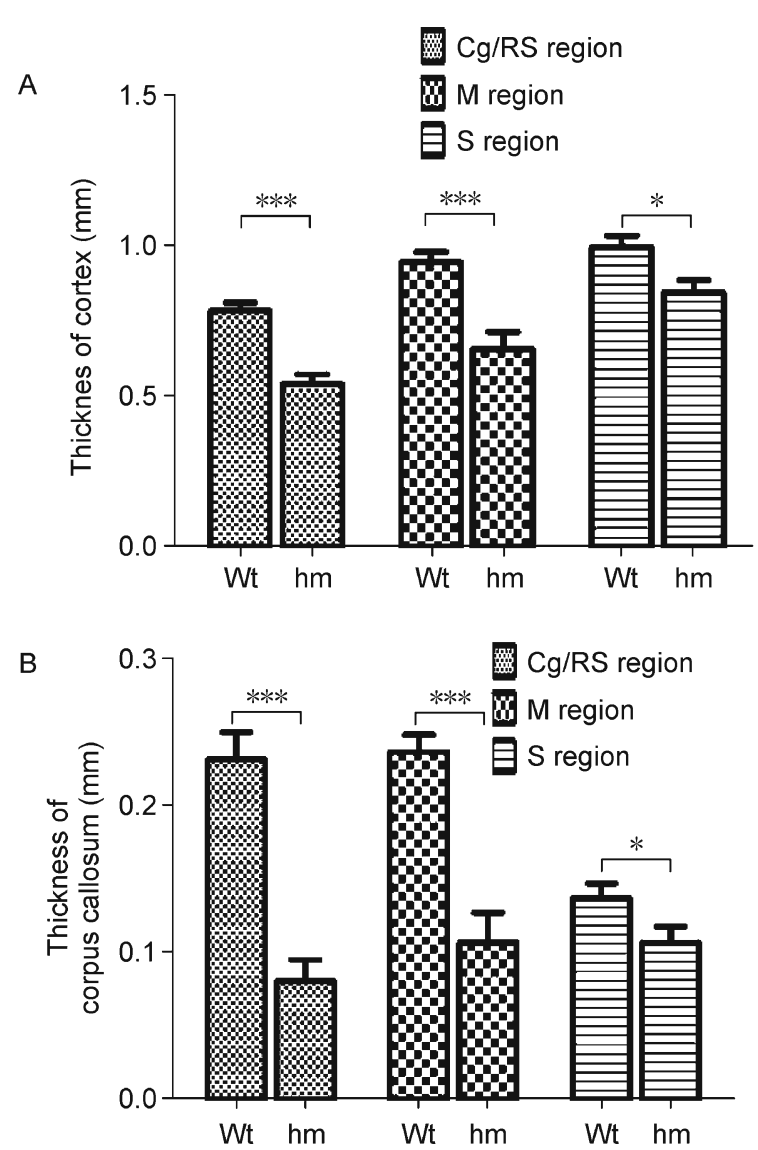

Figure 4. Malformation of the cerebral cortex and corpus callosum in homozygous Dscam ${ }^{\text {del17 }}$ mutant mice. (A) Comparison of the thickness of different cortical regions. The cortical thickness in cingulate/retrosplenial regions (Cg/RS) $(n=$ $20,0.54 \pm 0.03)$, motor $(\mathrm{M})(n=20,0.66 \pm 0.06)$ or sensory (S) cortices ( $n=20,0.85 \pm 0.04$ ) of homozygous $D$ scam ${ }^{\text {del17 }}$ mutant mice were significantly thinner than wild type mice $(\mathrm{Cg} / \mathrm{RS}: n=$ 13, $0.78 \pm 0.03 ; \mathrm{M}: n=13,0.95 \pm 0.03 ; \mathrm{S}: n=13,1.00 \pm 0.04)$ (means \pm SEM, two-way ANOVA, ${ }^{*} p<0.05,{ }^{* * *} p<0.001$ ). (B) Comparison of the corpus callosum thickness in different cortical regions. The thickness of the corpus callosum in $\mathrm{Cg} / \mathrm{RS}(n=20$, $0.08 \pm 0.02), \mathrm{M}(n=20,0.11 \pm 0.02)$ and $\mathrm{S}(n=20,0.11 \pm 0.01)$ regions of homozygous $D s c a m^{\text {del17 }}$ mutant mice were significantly thinner than wild type mice ( $\mathrm{Cg} / \mathrm{RS}: n=13,0.23 \pm 0.02$; M: $n=13,0.24 \pm 0.01$; S: $n=13,0.14 \pm 0.01$ ) (means \pm SEM, twoway ANOVA, $\left.{ }^{*} p<0.05,{ }^{* * *} p<0.001\right)$.

molecular pathogenic mechanisms underlying hydrocephalus caused by mutations in different genes.

\section{MATERIALS AND METHODS}

\section{Mouse strains}

$D s c a m^{\text {del17 }}$ mice were purchased form the Jackson Laboratory (Bar Harbor, ME). Dscam del17 homozygotes in C57BL/6 background die after birth. The experiments described in this study were carried out in the C57/BALB congenic background. Littermates of experimental mutant mice were used as controls. All experimental procedures were carried out following institutional and NIH guidelines.

\section{Genotyping}

$D s c a m^{\text {del17 }}$ mice were genotyped using the primers $D s c a m^{\text {del17}} \mathrm{F}$ (CTTTGCGCGTTATGATCCT) and Dscam de/17R (GTGGTGTCGATACTGATG) (Fuerst et al., 2008). Tail biopsies were performed and genomic DNAs extracted for genotyping. The PCR reactions were carried out as follows: $94^{\circ} \mathrm{C}$ for $5 \mathrm{~min}$, followed by 35 cycles of $94^{\circ} \mathrm{C}$ for $30 \mathrm{~s}, 60^{\circ} \mathrm{C}$ for $30 \mathrm{~s}$ and $72^{\circ} \mathrm{C}$ for $30 \mathrm{~s}$, and finally by extension at $72^{\circ} \mathrm{C}$ for $10 \mathrm{~min}$.

\section{Nissl staining}

At 2 months, animals were anesthetized and perfused with PBS, followed by pre-cold PBS containing 4\% paraformaldehyde. Brains were harvested and postfixed in perfusion solution overnight, followed by $15 \%$ sucrose in PBS overnight at $4^{\circ} \mathrm{C}$, and then by $30 \%$ sucrose in PBS overnight at $4^{\circ} \mathrm{C}$ and frozen in OCT media. $20 \mu \mathrm{m}$ sections were cut using a cryostat following published protocol (Frankin and Paxinos, 1997). Sections were stained in $0.1 \%$ crystal violet, and then washed in Milli-Q water for $3 \times 5 \mathrm{~min}$. The sections were destained in $95 \%$ ethanol for 5-10 min, followed by dehydration in $100 \%$ ethanol for $5 \mathrm{~min}$, and cleaning in Xylene for 3 times $(5 \mathrm{~min}$ each). Sections were mounted with resinene and stored at room temperature until imaging.

\section{Accelerated rotarod assay}

The accelerated rotarod is electronically controlled and started by manual switches and stopped automatically when all the mice fall off on the bottom of the apparatus. It consists of five plastic rods with a knurled surface for the mice to grip and flanges on both sides to confine the mouse on its own rod. The distance between the top of the rod and the bottom of the apparatus is $35 \mathrm{~cm}$. The rotarod can accelerate from $5 \mathrm{rpm}$ to $40 \mathrm{rpm}$ within $2 \mathrm{~min}$. Mice were placed on the rod in such a way that they were allowed to walk forward. After starting the apparatus, the speed and time at which the individual mouse fell off were recorded. Each mouse received three trials per session and the mean time was taken. For rotarod training, mice were tested in 8 trials, with four trials per day with a 45-60 min inter-trial interval over 2 days. The last trial was performed to test whether mice improved after rotarod training.

\section{Measurements of thickness of the cortex and corpus callosum}

Serial sections of $20 \mu \mathrm{m}$ in thickness were prepared with coronal sections spanning from the rostral to caudal parts of the brains (Frankin and Paxinos, 1997). The sections were stained with crystal violet. The ventricle area on each section and area of each section were measured by Image $\mathrm{J}$, respectively. To calculate the ventricle and brain volumes, the area from each serial section was estimated and multiplied by the section thickness. The calculated volumes, therefore, were a summation of all of areas in the serial sections multiplied by the thickness. The thickness of the cortex and corpus 
callosum were measured on the same Nissl-stained serial sections and measured by NeuroJ along the direction of neuronal projection (in micrometers). Each cortex and corpus callosum was measured three times.

\section{ACKNOWLEDGEMENTS}

We thank members of the Wu lab for helpful suggestions, stimulating discussions and technical assistance. The work is supported by grants from the Ministry of Science and Technology of China (Nos. 2009CB825402 and 2010CB529603) and National Natural Science Foundation of China (Grant No. 30900845).

Supplementary video on impaired locomotive function in homozygous $D$ scam del17 mutant mice is available in the online version of this article at http://dx.doi.org/10.1007/s13238-011-1072-8 and is accessible for authorized users.

\section{REFERENCES}

Agarwala, K.L., Ganesh, S., Suzuki, T., Akagi, T., Kaneko, K., Amano, K., Tsutsumi, Y., Yamaguchi, K., Hashikawa, T., and Yamakawa, K. (2001). Dscam is associated with axonal and dendritic features of neuronal cells. J Neurosci Res 66, 337-346.

Agarwala, K.L., Nakamura, S., Tsutsumi, Y., and Yamakawa, K. (2000). Down syndrome cell adhesion molecule DSCAM mediates homophilic intercellular adhesion. Brain Res Mol Brain Res 79, 118-126.

Amano, K., Fujii, M., Arata, S., Tojima, T., Ogawa, M., Morita, N., Shimohata, A., Furuichi, T., Itohara, S., Kamiguchi, H., et al. (2009). DSCAM deficiency causes loss of pre-inspiratory neuron synchroneity and perinatal death. J Neurosci 29, 2984-2996.

Barlow, G.M., Lyons, G.E., Richardson, J.A., Sarnat, H.B., and Korenberg, J.R. (2002a). DSCAM: an endogenous promoter drives expression in the developing CNS and neural crest. Biochem Biophys Res Commun 299, 1-6.

Barlow, G.M., Micales, B., Chen, X.N., Lyons, G.E., and Korenberg, J. R. (2002b). Mammalian DSCAMs: roles in the development of the spinal cord, cortex, and cerebellum? Biochem Biophys Res Commun 293, 881-891.

Basel-Vanagaite, L., Straussberg, R., Friez, M.J., Inbar, D., Korenreich, L., Shohat, M., and Schwartz, C.E. (2006). Expanding the phenotypic spectrum of L1CAM-associated disease. Clin Genet 69, 414-419.

Bertolin, C., Boaretto, F., Barbon, G., Salviati, L., Lapi, E., Divizia, M. T., Garavelli, L., Occhi, G., Vazza, G., and Mostacciuolo, M.L. (2010). Novel mutations in the L1CAM gene support the complexity of L1 syndrome. J Neurol Sci 294, 124-126.

Brites, D., McTaggart, S., Morris, K., Anderson, J., Thomas, K., Colson, I., Fabbro, T., Little, T.J., Ebert, D., and Du Pasquier, L. (2008). The Dscam homologue of the crustacean Daphnia is diversified by alternative splicing like in insects. Mol Biol Evol 25, 1429-1439.

Del Bigio, M.R. (2010). Ependymal cells: biology and pathology. Acta Neuropathol 119, 55-73.

Frankin, K.B.J., and Paxinos, G. (1997). The mouse brain in stereotaxic coordinates. New York: Academic.

Fuerst, P.G., Bruce, F., Tian, M., Wei, W., Elstrott, J., Feller, M.B., Erskine, L., Singer, J.H., and Burgess, R.W. (2009). DSCAM and DSCAML1 function in self-avoidance in multiple cell types in the developing mouse retina. Neuron 64, 484-497.

Fuerst, P.G., Koizumi, A., Masland, R.H., and Burgess, R.W. (2008). Neurite arborization and mosaic spacing in the mouse retina require DSCAM. Nature 451, 470-474.

Hattori, D., Demir, E., Kim, H.W., Viragh, E., Zipursky, S.L., and Dickson, B.J. (2007). Dscam diversity is essential for neuronal wiring and self-recognition. Nature 449, 223-227.

Haverkamp, F., Wölfle, J., Aretz, M., Krämer, A., Höhmann, B., Fahnenstich, H., and Zerres, K. (1999). Congenital hydrocephalus internus and aqueduct stenosis: aetiology and implications for genetic counselling. Eur J Pediatr 158, 474-478.

Hughes, M.E., Bortnick, R., Tsubouchi, A., Bäumer, P., Kondo, M., Uemura, T., and Schmucker, D. (2007). Homophilic Dscam interactions control complex dendrite morphogenesis. Neuron 54, 417-427.

Hummel, T., Vasconcelos, M.L., Clemens, J.C., Fishilevich, Y., Vosshall, L.B., and Zipursky, S.L. (2003). Axonal targeting of olfactory receptor neurons in Drosophila is controlled by Dscam. Neuron 37, 221-231.

Jackson, S.R., Guner, Y.S., Woo, R., Randolph, L.M., Ford, H., and Shin, C.E. (2009). L1CAM mutation in association with X-linked hydrocephalus and Hirschsprung's disease. Pediatr Surg Int 25, 823-825.

Jones, B.J., and Roberts, D.J. (1968). The quantiative measurement of motor inco-ordination in naive mice using an acelerating rotarod. J Pharm Pharmacol 20, 302-304.

Jouet, M., Rosenthal, A., MacFarlane, J., Kenwrick, S., and Donnai, D. (1993). A missense mutation confirms the L1 defect in X-linked hydrocephalus (HSAS). Nat Genet 4, 331.

Liebau, M.C., Gal, A., Superti-Furga, A., Omran, H., and Pohl, M. (2007). L1CAM mutation in a boy with hydrocephalus and duplex kidneys. Pediatr Nephrol 22, 1058-1061.

Liu, G., Li, W., Wang, L., Kar, A., Guan, K.L., Rao, Y., and Wu, J.Y. (2009). DSCAM functions as a netrin receptor in commissural axon pathfinding. Proc Natl Acad Sci U S A 106, 2951-2956.

Ly, A., Nikolaev, A., Suresh, G., Zheng, Y., Tessier-Lavigne, M., and Stein, E. (2008). DSCAM is a netrin receptor that collaborates with DCC in mediating turning responses to netrin-1. Cell 133, 1241-1254.

Matthews, B.J., Kim, M.E., Flanagan, J.J., Hattori, D., Clemens, J.C., Zipursky, S.L., and Grueber, W.B. (2007). Dendrite self-avoidance is controlled by Dscam. Cell 129, 593-604.

Meijers, R., Puettmann-Holgado, R., Skiniotis, G., Liu, J.H., Walz, T., Wang, J.H., and Schmucker, D. (2007). Structural basis of Dscam isoform specificity. Nature 449, 487-491.

Nakamura, Y., Lee, S., Haddox, C.L., Weaver, E.J., and Lemmon, V.P. (2010). Role of the cytoplasmic domain of the L1 cell adhesion molecule in brain development. J Comp Neurol 518, 1113-1132.

Schäfer, M.K., and Altevogt, P. (2010). L1CAM malfunction in the nervous system and human carcinomas. Cell Mol Life Sci 67, 2425-2437.

Schmucker, D., Clemens, J.C., Shu, H., Worby, C.A., Xiao, J., Muda, M., Dixon, J.E., and Zipursky, S.L. (2000). Drosophila Dscam is an axon guidance receptor exhibiting extraordinary molecular diversity. Cell 101, 671-684.

Soba, P., Zhu, S., Emoto, K., Younger, S., Yang, S.J., Yu, H.H., Lee, T., Jan, L.Y., and Jan, Y.N. (2007). Drosophila sensory neurons require Dscam for dendritic self-avoidance and proper dendritic 
field organization. Neuron 54, 403-416.

Tapanes-Castillo, A., Weaver, E.J., Smith, R.P., Kamei, Y., Caspary, T., Hamilton-Nelson, K.L., Slifer, S.H., Martin, E.R., Bixby, J.L., and Lemmon, V.P. (2010). A modifier locus on chromosome 5 contributes to $\mathrm{L} 1$ cell adhesion molecule $\mathrm{X}$-linked hydrocephalus in mice. Neurogenetics 11, 53-71.

Vos, Y.J., and Hofstra, R.M. (2010). An updated and upgraded L1CAM mutation database. Hum Mutat 31, E1102-E1109.

Wang, J., Zugates, C.T., Liang, I.H., Lee, C.H., and Lee, T. (2002). Drosophila Dscam is required for divergent segregation of sister branches and suppresses ectopic bifurcation of axons. Neuron 33, 559-571.

Wilson, P.L., Kattman, B.B., Mulvihill, J.J., Li, S., Wilkins, J., Wagner, A.F., and Goodman, J.R. (2009). Prenatal identification of a novel R937P L1CAM missense mutation. Genet Test Mol Biomarkers 13, 515-519.

Wojtowicz, W.M., Flanagan, J.J., Millard, S.S., Zipursky, S.L., and
Clemens, J.C. (2004). Alternative splicing of Drosophila Dscam generates axon guidance receptors that exhibit isoform-specific homophilic binding. Cell 118, 619-633.

Wojtowicz, W.M., Wu, W., Andre, I., Qian, B., Baker, D., and Zipursky, S.L. (2007). A vast repertoire of Dscam binding specificities arises from modular interactions of variable lg domains. Cell 130, 1134-1145.

Yamagata, M., Sanes, J.R. (2010). Synaptic localization and function of Sidekick recognition molecules require MAGI scaffolding proteins. J Neurosci 30, 3579-3588.

Yamakawa, K., Huot, Y.K., Haendelt, M.A., Hubert, R., Chen, X.N., Lyons, G.E., and Korenberg, J.R. (1998). DSCAM: a novel member of the immunoglobulin superfamily maps in a Down syndrome region and is involved in the development of the nervous system. Hum Mol Genet 7, 227-237.

Zhang, J., Williams, M.A., and Rigamonti, D. (2006). Genetics of human hydrocephalus. J Neurol 253, 1255-1266. 